\title{
Viele Schlaganfallpatienten sind nicht ausreichend antikoaguliert
}

Fragestellung: Welche vorbestehende antithrombotische Therapie hatten Patienten mit bekanntem Vorhofflimmern, die einen akuten ischämischen Insult erleiden?

Hintergrund: Vorhofflimmern ist ein wichtiger Risikofaktor für den ischämischen Insult und erhöht das Schlaganfallrisiko um den Faktor 5. Das Schlaganfallrisiko bei Patienten mit Vorhofflimmern kann durch eine konsequente Antikoagulation um $60-70 \%$ reduziert werden. In den vergangenen zehn Jahren zeigte eine Reihe von Studien, dass allerdings der Löwenanteil von Patienten mit Vorhofflimmern, die einen ischämischen Insult erleiden, zum Zeitpunkt des Schlaganfalls nicht oder nur unzureichend behandelt werden. Dies sollte jetzt in den USA in dem großen Schlaganfallregister "Get with the Guidelines“ untersucht werden.

Patienten und Methodik: Bei der vorliegenden Untersuchung handelt es sich um eine retrospektive Analyse von 94.474 Patienten mit akutem ischämi-

Xian Y, O’Brien EC, Liang L et al. Association of preceding antithrombotic treatment with acute ischemic stroke severity and in-hospital outcomes among patients with atrial fibrillation JAMA 2017; 317: 1057-67 schem Insult und zuvor bekanntem Vorhofflimmern. Die Patienten wurden zwischen Oktober 2012 und März 2015 in 1.622 Krankenhäusern in den USA wegen eines Schlaganfalls stationär aufgenommen. Dort wurde die antithrombotische Therapie zum Zeitpunkt des Schlaganfalls erfasst. Untersucht wurden zudem die Schwere des Schlaganfalls gemessen mit der NIHSS und die Sterblichkeit während des Krankenhausaufenthalts.

Ergebnisse: Die 94.474 Patienten waren im Mittel 80 Jahre alt und $57 \%$ waren Frauen. Von diesen Patienten waren 30,3\% unbehandelt, $40 \%$ erhielten Thrombozytenfunktionshemmer in Monotherapie und 13,5\% waren mit Vitamin-K-Antagonisten (VKA) antikoaguliert, hatten allerdings zum Zeitpunkt des Schlaganfalls eine INR von $<2$. Ausreichend antikoaguliert waren lediglich 7,6\% der Patienten, die Warfarin erhielten und eine INR von $>2$ hatten sowie $8,8 \%$ Patienten, die mit einem NichtVKA-Antikoagulans (NOAK) behandelt worden waren.

Patienten, die unter therapeutischer Antikoagulation einen Schlaganfall erlitten, hatten eine geringere Schwere des Schlaganfalls und eine um $3 \%$ reduzierte Mortalität (6,4\% vs. 9,3\%). Die Odds Ratio für eine geringere Wahrscheinlichkeit eines mittelschweren oder schweren Schlaganfalls unter therapeutischer Antikoagulation betrug 0,56. Für die Sterblichkeit im Krankenhaus betrug die Odds Ratio 0,75.

Schlussfolgerungen: Nur ein geringer Anteil von Patienten mit bekanntem Vorhofflimmern ist zum Zeitpunkt des ischämischen Insults ausreichend antikoaguliert. Patienten mit therapeutischer Antikoagulation haben eine geringere Schlaganfallschwere und versterben seltener während des Klinikaufenthalts.

\section{- Kommentar von Hans-Christoph Diener, Essen}

\section{Anstrengungen bei der Antikoagulation weiter erhöhen}

Die Daten aus den USA sind verheerend. Sie zeigen weiterhin, dass der Großteil von Patienten mit bekanntem Vorhofflimmern zum Zeitpunkt ihres ischämischen Insults entweder unbehandelt sind, lediglich einen Thrombozytenfunktionshemmer erhalten, der nur eine geringe Wirksamkeit in der Schlaganfallprävention hat, oder wenn sie mit VKA behandelt

\section{Weitere Infos auf springermedizin.de}

Antikoagulation des adipösen Patienten

Der Artikel informiert über Dosierungsprobleme von Antikoagulanzien bei adipösen Menschen. Sie finden ihn, indem Sie den Titel in die Suche eingeben. sind, eine subtherapeutische INR aufweisen. Dies hat offenbar nicht nur Auswirkungen auf die Häufigkeit von Schlaganfällen, sondern auch auf die Schwere des Schlaganfalls und die Sterblichkeit im Krankenhaus. Für die klinische Realität bedeutet dies, dass noch deutlich mehr Anstrengungen unternommen werden müssen, um Patienten mit bekanntem Vorhofflimmern ausreichend zu antikoagulieren.

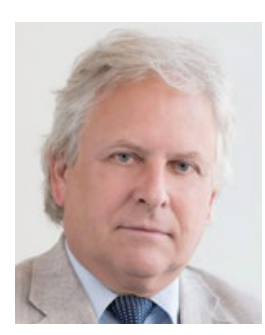

Prof. Dr. med. Hans-Christoph Diener, Essen

Seniorprofessor für klinische Neurowissenschaften, Universitätsklinikum Essen E-Mail:h.diener@uni-essen.de 